\title{
RANS Simulation of HAWT immersed on ABL flow under neutral and stratified conditions using OpenFOAM
}

\author{
Mohammed Nadjib Hamlaoui ${ }^{1}$, Arezki Smaili ${ }^{1}$, Hachimi Fellouah ${ }^{2 *}$ \\ ${ }^{1}$ Laboratoire de Génie Mécanique et Développement, Ecole Nationale Polytechnique, Alger, Algérie \\ ${ }^{2}$ Department of Mechanical Engineering, Universitée de Sherbrooke, Quebec, Canada \\ *Hachimi.Fellouah@usherbrooke.ca
}

\begin{abstract}
This paper proposes numerical method for aerodynamic performance predictions of Horizontal Axis Wind Turbines (HAWT) immersed on Atmospheric Boundary Layer (ABL) flows under unstable, stable and neutral conditions. The flow field has been described using the three-dimensional RANS equations complemented by the $k-\varepsilon$ turbulence model with modified constants corresponding to ABL flows. The density variation has been introduced into the momentum equation using the Boussinesq approximation and appropriate buoyancy term has been included. The equation of energy based on potential temperature has been implemented and solved to take into account the effect of temperature on the flow field (turbulence). The ABL profiles ( $k, \varepsilon$, Velocity and Temperature) under neutral and stratified conditions has been implemented into OpenFOAM based on MoninObukhov similarity. The flow over the fetch has been calculated through an in-house solver (ABLSolversimpleFoam) developed with OpenFOAM for steady-state turbulent flows. Comparison between the solutions of the ABL proposed by the present method and the empirical relations based on Monin-Obukhov similarity theory has been carried out by giving good accuracy. The immersed HAWT has been modeled using the actuator disc approach where the swept surface of the rotor is replaced by surface forces that act upon the incoming flow according to the Blade Element theory. The Blade Element theory uses the two dimensional aerodynamic coefficients which produce under predictions of the power output at high incoming wind speeds. Du and Selig stall delay model has been implemented to take into account the effect of rotation. The obtained results have indicated that the velocity defect in the wake of a wind turbine is more significant in unstable than in stable thermal stability conditions. Consequently, it involves a significant variation of the performances of a wind turbine placed in the direct wake of another resulting on a power output drop of $37.64 \%$ and $13.67 \%$ respectively.
\end{abstract}

